\title{
Artefact
}

Techniques, histoire et sciences humaines

$6 \mid 2017$

Histoire et archéologie

\section{Une collection exceptionnelle : les textiles gallo- romains des Martres-de-Veyre (Puy-de-Dôme) conservés au musée Bargoin de Clermont-Ferrand}

Catherine Breniquet, Marie Bèche-Wittmann, Christine Bouilloc et Camille Gaumat

\section{(2) OpenEdition}

1 Journals

Édition électronique

URL : http://journals.openedition.org/artefact/902

DOI : 10.4000/artefact.902

ISSN : 2606-9245

Éditeur :

Association Artefact. Techniques histoire et sciences humaines, Presses universitaires du Midi

Édition imprimée

Pagination : 197-207

ISBN : 978-2-7535-7305-5

ISSN : 2273-0753

\section{Référence électronique}

Catherine Breniquet, Marie Bèche-Wittmann, Christine Bouilloc et Camille Gaumat, « Une collection exceptionnelle : les textiles gallo-romains des Martres-de-Veyre (Puy-de-Dôme) conservés au musée Bargoin de Clermont-Ferrand », Artefact [En ligne], 6 | 2017, mis en ligne le 31 mai 2018, consulté le 15 septembre 2020. URL : http://journals.openedition.org/artefact/902

\section{(c) (i) (9)}

Artefact, Techniques, histoire et sciences humaines est mise à disposition selon les termes de la Licence Creative Commons Attribution - Pas d'Utilisation Commerciale - Pas de Modification 4.0 International. 


\section{Une collection exceptionnelle : les textiles gallo-romains des Martres- de-Veyre (Puy-de-Dôme) conservés au musée Bargoin de Clermont-Ferrand}

Catherine BReniQuet, Marie Bèche-WittMann, Christine Boullloc*, Camille GAUMAT ${ }^{* *}$

Depuis son inauguration en 1903, le musée Bargoin de Clermont-Ferrand abrite une collection de textiles galloromains exceptionnels par leur état de conservation. Le grand préhistorien Joseph Déchelette disait, en 1911, à leur propos : «Il me semble qu'il serait nécessaire de publier de bonnes phototypies de chacun de ces échantillons. On n'a pas tous les jours à sa disposition des tissus de l'époque romaine et il faudrait tirer de ces documents tout le parti possible ${ }^{1}$. " Ce fut partiellement chose faite en 1923. Cette collection, dont certaines pièces sont mondialement connues, provient du site des Martres-de-Veyre, situé à une quinzaine de kilomètres de ClermontFerrand, plus spécifiquement d'une nécropole gallo-romaine découverte fortuitement en 1851 et explorée depuis à plusieurs reprises, en 1893 et 1922.
Bien que plusieurs érudits locaux aient rapporté les premières découvertes et tenté d'en encadrer une exploration assez peu méthodique ${ }^{2}$, le grand artisan de la valorisation de ces collections, comme de la reprise des fouilles de la nécropole, fut Auguste Audollent (18641943), directeur du musée Bargoin, professeur et doyen de la Faculté des lettres de l'université clermontoise. Arrivé à Clermont-Ferrand en 1893, Audollent, ancien membre de l'École française de Rome et spécialiste reconnu de la Carthage romaine, fut immédiatement confronté à une archéologie auvergnate qui lui permit de conjuguer avec bonheur son intérêt scientifique pour le monde romain tout en s'impliquant localement grâce aux fouilles du temple de Mercure, au sommet du Puy de Dôme, et aux Martres-de-Veyre. Tous ceux qui

*. Catherine Breniquet est professeur d'histoire de l'art et archéologie antiques à l'université ClermontAuvergne. Archéologue orientaliste, elle a été pensionnaire scientifique à la Délégation archéologique française en Iraq (DAFIq). À la suite des événements qui ont frappé le Proche-Orient dans les années 1990, elle a réorienté ses activités de recherche vers l'archéologie du textile (Essai sur le tissage en Mésopotamie, des premières sociétés sédentaires au milieu du III millénaire avant J.-C., de Boccard, Paris, 2008). Elle étudie également des textiles venant d'autres civilisations et elle développe des perspectives anthropologiques.

**. Musée Bargoin, Clermont-Métropole. 
l'approchèrent dirent qu'il n'était pas qu'homme de cabinet comme ses fonctions académiques pouvaient le laisser croire; il était aussi un homme de terrain, au fait des dernières avancées méthodologiques de l'archéologie, et au cœur d'un vaste réseau scientifique national, voire européen. C'est lui qui rassembla les principales informations sur la nécropole gallo-romaine des Martres-de-Veyre et les publia dans des revues scientifiques très diffusées alors. Un programme d'étude et de valorisation de ces collections est actuellement en cours ${ }^{3}$, associé à un important travail de récolement, également en cours, effectué par le musée. L'ensemble permet la redécouverte d'un corpus exceptionnel constitué de plus de quatre-vingts fragments textiles.

\section{Le contexte des découvertes}

Dès la première moitié du XIx ${ }^{e}$ siècle, le petit village des Martres-de-Veyre, implanté au pied du plateau de Corent, dans une boucle de l'Allier, est identifié comme « une terre féconde pour l'archéo$\operatorname{logie}^{4} »$. Plusieurs découvertes d'époque gauloise ou romaine ont déjà attiré l'attention sur lui : un casque en bronze " italo-étrusque » et d'importants lots de céramique sigillée. Mais c'est en 1851 que des ouvriers de la tuilerie Vazeilhes, occupés à extraire de l'argile au lieu-dit " Le Lot» (ou " Le Lod", voire encore "Le Lau »), découvrent fortuitement les inhumations gallo-romaines. Une première tombe est fouillée à la hâte. Une seconde défraye la chronique; elle livre, dans un cercueil de bois, les restes bien identifiables d'une jeune femme couchée sur le ventre, à l'évidence habillée de plusieurs pièces vestimentaires superposées, et chaussée d'une paire de sandales de cuir à semelle de liège. La tête de cette femme reposait sur une couverture quadrillée polychrome (un sergé de type " tartan " gaulois), tandis que le haut de son corps était protégé par une autre couverture évoquant " la toison $d^{\prime}$ un mouton ${ }^{5}$ ». La tombe livre également différents objets, céramique, verre, dépôts alimentaires, etc., non inventoriés de façon systématique au moment des découvertes et rapidement dispersés, certains ayant été récupérés par des amateurs locaux anonymes. La découverte, relayée par la presse locale, suscite le scepticisme : comment concilier une datation antique de cet ensemble avec une si bonne conservation des matériaux organiques? Finalement, JeanBaptiste Bouillet, directeur du musée de Clermont-Ferrand, alors installé dans l'ancien hôpital des Charitains, se range à l'évidence : la poterie permet de dater cet ensemble de l'époque gallo-romaine $\left(\mathrm{I}^{\mathrm{er}}-\mathrm{II}{ }^{\mathrm{e}}\right.$ siècles apr. J.-C. $\left.{ }^{6}\right)$. Il sauve aussi du désastre quelques pièces qui sont exposées et publiées ${ }^{7}$.

Plusieurs décennies se passent avant qu'une autre série de tombes soit mise au jour. En 1893, année où Audollent arrive à Clermont-Ferrand, des urnes cinéraires sont exhumées dans un terrain attenant, ainsi que d'autres inhumations livrant ou non des cercueils de bois et des défunts habillés ${ }^{8}$, associés à un mobilier désor- 
mais habituel (poteries gallo-romaines, monnaies, fioles de verre, pyxides en bois, corbeilles, offrandes alimentaires ou végétales en proportions variables ${ }^{9}$, etc.). C'est à l'une de ces inhumations, la tombe $\mathrm{D}$ - les tombes sont affectées d'une lettre (A et $B$ pour les tombes découvertes en 1851, C à F pour celles de 1893) -, que se rapportent les pièces maîtresses des collections textiles des Martres-de-Veyre, la fameuse tunique et sa " ceinture ", associées à des chausses, acquises en 1893 par le musée de Clermont-Ferrand (fig. 24, cahier couleur). Ce sont en réalité au moins quatre nouvelles inhumations qui viennent compléter les deux dégagées en 1851 et qui livrent elles aussi des vestiges organiques de même nature, en excellent état de conservation, parmi lesquels des vêtements, chaussures, textiles divers : un homme couché en pleine terre (tombe $\mathrm{C}$ ), deux femmes (tombes $\mathrm{D}$ et $\mathrm{E}$ ) et une petite fille (tombe F). Toutes les découvertes confondues contribuent à faire reconnaître l'intérêt du site et attirent les savants au musée qu'administre désormais Audollent. Parmi eux, on trouve Joseph Déchelette, originaire de la ville toute proche de Roanne, avec qui Audollent entretient une correspondance substantielle. Celui-ci est intéressé par les vestiges qui documentent les techniques industrielles de l'Antiquité, céramiques, mais aussi textiles, rarement conservés, surtout dans un tel état!

\section{Les publications des découvertes}

Quelques années passent encore tandis qu'Audollent entreprend un vaste travail d'inventaire de ces découvertes qu'il présente devant l'Académie des inscriptions et belles-lettres, en 1921, grâce à l'intermédiaire de Camille Jullian; il le publie auprès de cette même académie $^{10}$. La plupart des chercheurs se réfèrent encore aujourd'hui à cette publication qui propose une mise en ordre bienvenue des découvertes. Les tombes sont décrites aussi précisément que possible, malgré l'apparent désordre des premières explorations ${ }^{11}$. Un inventaire raisonné et systématique de l'ensemble du mobilier est également proposé. Cette publication est pourtant partielle. Elle est sous-illustrée (au grand dam de son auteur ${ }^{12}$ ), se contredit parfois en raison de sa rédaction a posteriori par quelqu'un qui n'a pas pris part aux fouilles, mais surtout elle ne prend en compte ni les tombes à crémation contemporaines trouvées dans les mêmes terrains, ni les fouilles menées par Audollent lui-même en 1922-1923 ${ }^{13}$. Bien qu'encouragées par la Société française des fouilles archéologiques et la municipalité de ClermontFerrand, ces dernières s'avèrent plus délicates en raison de l'emploi du temps académique d'Audollent, contraint de stopper le chantier pour cause d'examens universitaires, mais aussi en raison $\mathrm{du}$ terrain qui livre sans surprise inhumations et crémations mêlées, et d'une trésorerie problématique. Leurs résultats sont aussi plus austères. La moisson est moins spectaculaire que prévue. Une tombe (I), par ailleurs la seule qui ait été photographiée de cet ensemble, livre des 


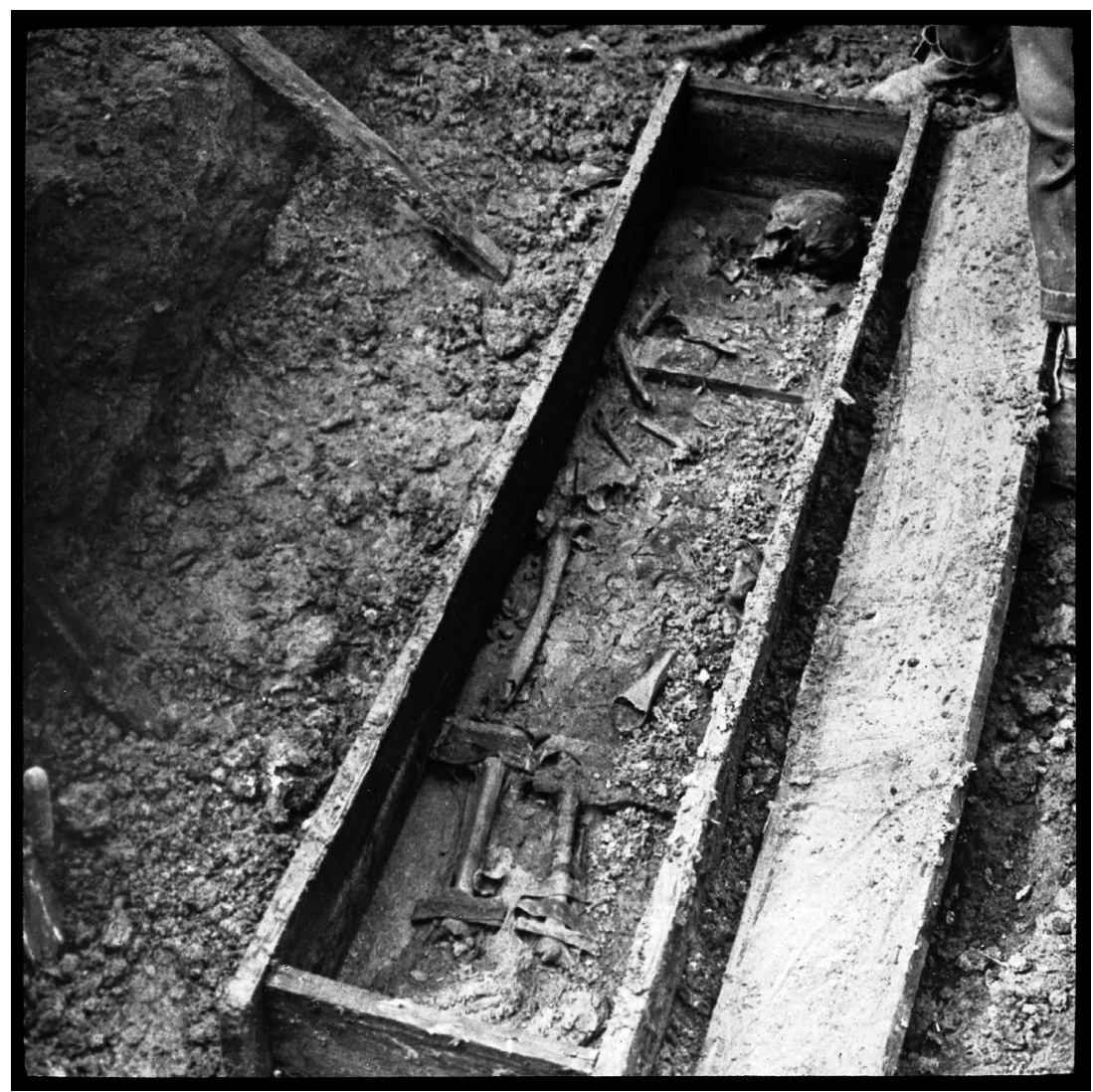

Figure 1. - Tombe I, fouilles Audollent, 1922-1923 @ Musée Bargoin, Clermont-Métropole.

restes textiles identifiables, des bandes molletières en l'occurrence, des restes de cheveux et des chaussures (fig. 1). Le cercueil en sapin intact est emporté au musée et exposé sans dommage dans les salles jusqu'à la refonte du département archéologique en 2016. Deux autres coffres en bois, vestiges d'inhumations mal préservées et trouvées à proximité, auraient également été rapatriés. L'un d'eux est un petit coffre qui renfermait les restes d'un enfant trouvé à proximité de la tombe I, mêlés à une « étoffe sombre ».

Fort de son réseau scientifique personnel, Audollent est aussi sollicité pour diffuser et vulgariser les résultats des premières découvertes. Il donne une conférence au musée Guimet en 1924, contribuant ainsi à faire connaître le site et ses collections remarquables, et publie deux articles outre-Manche, l'un dans la prestigieuse revue anglo-saxonne Man, l'autre dans Notes and Queries ${ }^{14}$. La publication de 1923 et ces deux dernières alimentent encore la bibliographie anglaise sur le costume gallo-romain. 


\section{Un peu d'archéologie du textile}

Les collections textiles, exceptionnelles par leur nombre et leur état de conservation, bénéficient dès la publication de 1922 d'une étude technique confiée à Charles Pagès, professeur de tissage à l'École de commerce de Lyon. Aiguillonné par Déchelette, Audollent lance l'étude et sa publication intégrale, à la suite de son propre mémoire sur les tombes à inhumation des Martres-deVeyre, malgré le décès de Pagès en $1921^{15}$. Il s'agit là d'une étude pionnière dans le champ de l'archéologie gallo-romaine des débuts du $\mathrm{xx}^{\mathrm{e}}$ siècle. Audollent fait parvenir à Pagès une série de treize échantillons de quelques centimètres carrés, provenant des tombes $\mathrm{A}, \mathrm{D}$ et $\mathrm{E}$. Ceux-ci portent les traces de découpe au ciseau qu'Audollent lui-même infligea à la collection! Tant pour les préserver d'une altération potentielle que pour en faciliter l'observation, Pagès monta ces échantillons sur des cartons forts, rassemblés dans une boîte que le musée conserve et annotés de sa main (fig. 25, cahier couleur).

L'archéologie du textile représente une discipline peu pratiquée en France, à la fois en raison de la difficulté (toute relative par ailleurs) à disposer d'un matériel d'étude bien conservé et de traditions de recherche qui valorisent d'autres catégories d'artefacts. On s'accorde toutefois à penser désormais que le potentiel d'étude des textiles anciens est considérable et que ceux-ci sont beaucoup plus représentés qu'on ne l'imagine. La difficulté réside en fait dans leur " traçage " archéologique et leur prise en compte comme artefacts, ainsi que dans le faible nombre des spécialistes disponibles.
Dans bien des cas, ce sont des fragments de petite taille que l'archéologie exhume. Ceux-ci proviennent fréquemment de contextes funéraires à cause du milieu particulier que constituent les sépultures et des progrès de l'archéologie funéraire, désormais attentive aux moindres vestiges. Mais c'est aussi le contact avec des objets métalliques piqués dans des tissus comme les fibules ou déposés sur eux (monnaies, armes) qui fournit des échantillons dans un état de minéralisation variable, mais pouvant autoriser l'identification des matériaux textiles. Les contextes de sécheresse extrême (Égypte, désert de Judée) ou humides (tourbières, tombes gelées, etc.) livrent quant à eux des exemplaires conservés "souples» et dans leur intégralité. Des empreintes de tissus sur des matériaux plastiques (argile, plâtre, bitume) complètent les découvertes.

D'un point de vue méthodologique, la discipline procède par observation et prise en compte des critères morphométriques des échantillons considérés, comme pour la plupart des études de matériel archéologique. Pagès en a largement jeté les bases sans le vouloir, s'inspirant de sa pratique de l'analyse des tissus en usage dans l'industrie textile ${ }^{16}$. Chacun des échantillons bénéficie d'une description technique systématique qui prend en compte deux grands paramètres : l'étude de la chaîne (les fils tendus sur le métier à tisser) et de la trame (fil mobile qui les solidarise grâce au passage de la navette) et de la " contexture ", c'est-à-dire l'armure (ou mode de croisement des fils) et la densité des fils de chaîne et de trame. 
L'identification de la chaîne et de la trame s'appuie sur des observations précises (présence de lisières, d'ourlets, de franges, etc.) permettant d'orienter le tissu dans son sens de fabrication. Une attention particulière est portée au matériau. Majoritairement, les textiles des Martres-de-Veyre sont en laine, mais d'autres matériaux, lin ou chanvre ${ }^{17}$, sont attestés de façon plus ponctuelle. Sur la foi d'informations glanées auprès des paysans de la commune et sans vérification possible, un érudit du $\mathrm{XIX}^{\mathrm{e}}$ siècle rapporte la présence d'une «étoffe tissue $\mathrm{d}^{\prime}$ or et de soie ${ }^{18}$ » dont il ne subsiste aucune trace et qui relève sans doute de l'imagination en raison du caractère modeste de ces tombes ${ }^{19}$. La structure de chaque fil est donnée (simple ou retors), sa torsion (" gauche " ou " droite " comme on disait à l'époque, soit dans le sens inverse des aiguilles d'une montre ou non, soit encore $\mathrm{Z}$ ou $\mathrm{S}$ selon les habitudes actuelles), et sa torsion approximative au mètre. Le spécialiste des techniques industrielles qu'était Pagès ajouta des éléments courants pour lui, mais peu fréquents en archéologie (en raison de l'état de conservation des textiles qui autorise rarement ces observations) : le titrage approximatif des

\section{Les pièces textiles}

La pièce maîtresse, mondialement connue, est une tunique provenant de la tombe D (fig. 24, cahier couleur), qui abritait le corps d'une jeune femme. La défunte était habillée d'une robe de laine brune sur laquelle des traces bleutées (plutôt dues au contact avec un objet fils, soit la longueur pour un poids, ici un kilogramme (ce que l'on assimilerait aujourd'hui au "Tex", qui est le poids en gramme pour 1000 mètres de fill), l'embuvage présumé (c'est-à-dire le retrait dans le sens chaîne lié au tissage lui-même) et partant le poids présumé. Dans un deuxième temps, l'étude de la contexture propose les données relatives à l'armure (toile ou sergé), à la réduction (nombre de fils et de duites, ou trames, au centimètre, rapports d'armure, etc.) et souligne la présence éventuelle d'éléments liés à la fabrication (lisières, ourlets, etc.). Des observations sur le parallélisme des fils de trame lui permettent de déduire des procédés ou gestes techniques (trame lancée «à la déroulée »). Les textiles des Martres-de-Veyre présentent souvent une couleur brune qui semble uniforme au premier abord, mais des observations de détail autorisent Pagès à identifier les pièces qui ont été teintes dans l'Antiquité. Enfin, ce sont les usages de ces textiles qui sont envisagés. Ceux des pièces conservées dans leur intégralité sont raisonnablement clairs (vêtements, couvertures, etc.). Pour les pièces fragmentaires, souvent arrachées à la hâte à leur contexte de découverte ${ }^{20}, c^{\prime}$ est une toute autre affaire...

métallique qu'à une volonté décorative ${ }^{21}$ ) ont pu être observées. Ce vêtement de belle ampleur $(1,25 \mathrm{~m}$ de hauteur pour $1,70 \mathrm{~m}$ de largeur, manches étendues) est composé de trois rectangles de tissu assemblés au moyen de coutures : le plus grand, plié aux épaules, forme le 
devant et le dos, deux autres constituent les manches. Le vêtement a été raccourci au moyen d'un pli cousu à grands points avec un fil blanc, au niveau de la taille ${ }^{22}$. La robe était associée à une longue bande frangée claire, elle-même en laine, agrémentée de tresses décoratives à plusieurs endroits, considérée comme étant une ceinture, alors qu'il s'agirait plutôt d'un strophion, une bande destinée à entourer la poitrine, selon l'hypothèse séduisante de Hero Granger-Taylor ${ }^{23}$. De grandes dimensions (4,30 m par 0,12 $\mathrm{m}$ ), élargie (déformée?) en son centre, celle-ci est rythmée de motifs tissés évoquant des tresses, disposés aux extrémités et au milieu (visible sur la fig. 24, cahier couleur). Ces deux pièces sont tissées en armure toile, avec des qualités de laine et des réductions différentes (toile 2.1 pour la tunique, toile à effet chaîne pour la ceinture). La laine utilisée pour la tunique a peut-être été peignée tant les fibres semblent parallèles entre elles et régulières. La panoplie de la défunte était complétée par des bas ou chausses en laine frangés, tissés en sergé, assemblés par couture, montant plus haut que les genoux et non ourlés, qui devaient être à l'origine maintenus par une jarretière ou un lien. L'un de ces bas est brodé de trois lettres blanches, PRI. Une paire de chaussons de laine, tissés en sergé et également assemblés par couture, est souvent attribuée à cet ensemble sans qu'il soit possible de le confirmer en raison de l'absence de mention afférente dans les inventaires dressés au moment des fouilles ${ }^{24}$. En revanche, des chaussures de cuir à semelles cloutées, d'une petite pointure, sont associées à cette sépulture.

Ce costume exceptionnel a été manipulé et exposé sans ménagement au musée dès qu'il y fit son entrée : sur mannequin, sur cintre, à plat. Aujourd'hui, il fait l'objet de plus d'égards, les diverses pièces n'étant ni exposées ensemble, ni même toutes sorties des réserves en raison de leur fragilité et de leur sensibilité à la lumière.

On déduit aisément de la publication qu'il existe une multitude de fragments textiles provenant des diverses tombes. Ceux-ci présentent l'heureuse particularité d'être de " grandes » dimensions, eu égard aux minuscules fragments que traque la plupart du temps l'archéologie, mais ils transparaissent mal dans la synthèse d'Audollent, car celui-ci a surtout insisté sur les fragments envoyés à Pagès, les autres étant encore « bruts de fouille » au moment de la rédaction du rapport. Ainsi, on sait moins qu'il existe une seconde tunique complète mais taillée en pièces, sans doute pour en faciliter le prélèvement lors de la fouille, peut-être associée à une seconde " ceinture ». Un premier inventaire et un essai d'assemblage des morceaux, inédits, ont été réalisés par Sophie Desrosiers en 1996 et 199725 , suite à une campagne de conservation-restauration effectuée par l'atelier de Béatrice Girault-Kurtzemann à Orange, en 1996-1997. Il serait prématuré de proposer un patron définitif de cette seconde tunique, en l'absence de vérifications actualisées, mais elle semble différer de la première au moins par la présence de clavi rouges encadrant l'encolure, par une construction et par un mode d'assemblage différent.

La provenance exacte de cette seconde tunique est loin d'être assurée. Audollent l'attribue à la tombe $\mathrm{A}$, découverte en 1851 et qui a livré d'autres fragments en tapisserie (fig. 26a, cahier couleur), mais 
les pièces restaurées depuis livrèrent à l'examen du verre pilé, non répertorié dans cette sépulture, mais attesté ailleurs, dans la tombe E, par exemple, l'autre tombe féminine dont la chevelure est aussi conservée ${ }^{26}$. Audollent n'était pas présent sur le terrain au moment des découvertes et les observations n'ont pas été systématiques. Il est donc extrêmement délicat de conclure en l'état actuel du dossier.

D'autres étoffes plus fragmentaires conservées au musée Bargoin proviennent des autres tombes galloromaines du site. Elles n'ont pas toujours de provenance claire en dépit des précisions données par Audollent : toiles de laine plus ou moins fines, parfois superposées, suggérant des vêtements enfilées les uns sur les autres, comme dans cette même tombe $A^{27}$, fragments de toile de lin ou de chanvre qui sont peut-être des chemises ou des sous-vêtements (tombes A et peut-être E) ou des restes de linceuls pliés (tombe F), pièces plus épaisses tissées en armure sergé dont une face semble grattée (manteau ou couverture, tombe A ou F) (fig. 26b, cahier couleur), châle frangé (tombe E) (fig. 27, cahier couleur), étoffe à carreaux (type tartan) tissée en armure sergé (tombe A) (fig. 26c, cahier couleur), etc. Toutes ne sont pas identifiées, ni même peut-être identifiables avec précision. Seule une étude comparative de l'ensemble des fragments autorisera des éléments de réponse. Certaines de ces pièces, les plus complètes, devraient permettre la réalisation de leur patron. De nombreuses observations techniques complémentaires (présence de lisières, points de couture, reprises, etc.) sont à venir. Les quelques échantillons de fils colorés qui ont été analysés ont révélé la présence de colorants d'origine végétale ${ }^{28}$.

De façon à compléter ce panorama, on mentionnera deux dernières catégories d'objets textiles : une petite quenouille à main, encore chargée d'une laine blanche à laquelle se mêlaient des brins de couleur bleu, rouge et jaunevert, aujourd'hui très altérés, trouvée dans la tombe $\mathrm{F}$ qui abritait le corps d'une petite fille, et deux corbeilles en vannerie (tombes $\mathrm{D}$ et $\mathrm{F}$ ) ${ }^{29}$ contenant des fruits secs. Enfin, ce sont au moins trois paires de chaussures qui complètent les ensembles vestimentaires : souliers de cuir de la tombe $\mathrm{D}$, sabots à semelle de liège de la tombe $\mathrm{A}$, sandales de bois garnies d'une semelle en peau de mouton de la tombe E.

Pour la plupart, ces pièces, tunique, ceinture et bas en tête, portent les marques du temps et ont donc été portées : repli cousu à grands points pour raccourcir la tunique, accrocs, traces de feutrage et d'usure en plusieurs endroits. Plusieurs fragments décolorés suggérent une longue utilisation et exposition à la lumière. Il serait sans doute risqué d'en tirer des conclusions immédiates et au premier degré sur les modes vestimentaires de la période gallo-romaine. Certes, la panoplie de la tombe $\mathrm{D}$ constitue le seul vêtement complet des provinces occidentales de l'empire romain, occupant ainsi un rang appréciable au milieu des pièces orientales, mais rien ne dit que cet ensemble est représentatif de la réalité passée. En effet, si l'on peut qualifier les inhumations des Martres-de-Veyre de sépultures habillées, nous ignorons tout des modalités de choix des vêtements (appartenant au défunt? à ses proches? habits quotidiens ou réservés aux grands 
jours?). À cet égard, la tunique, large et courte, conviendrait aussi bien à un homme qu'à une femme ${ }^{30} \ldots$ Des gestes rituels non pris en compte lors des premières fouilles viennent compliquer notre perception du costume. Ainsi en va-t-il de dépôts de pièces vestimentaires comme les chaussures. Les chaussures de cuir de la tombe $\mathrm{D}$, en raison de leur toute petite pointure $(23 \mathrm{~cm}$ de longueur, soit l'équivalent de notre 34), ne peuvent avoir été retrouvées enfilés aux pieds de la morte si celle-ci portait effectivement les chausses. Ces souliers étaient sans doute déposés dans la tombe.

L'ensemble des pièces textiles des Martres-de-Veyre constitue une collection rare à redécouvrir. La diversité technique dont les pièces témoignent (armures, jeux de fils simples ou retors, réduction ou contexture, teintures), montre une régularité de tissage ainsi que des effets décoratifs qui en font des productions soignées, sans être pour autant des pièces exceptionnelles pour l'époque. Bien qu'on ignore leur lieu de fabrication et les conditions dans lesquelles elles se retrouvèrent aux Martresde-Veyre ${ }^{31}$, elles éclairent l'artisanat du tissage et sa variété à l'époque galloromaine, répondant à une demande et des besoins spécifiques, les "modes » vestimentaires, plus complexes qu'on le croit généralement, de même que les pratiques funéraires, avec leur mise en scène. Cette diversité, associée au peu de données publiées, relativise sévèrement la plupart des tentatives de synthèse existantes. Quant à l'état de conservation exceptionnel de ces pièces, peut-être dû aux émanations de gaz carbonique liées à la présence de sources minérales dont le sous-sol des Martres-de-Veyre est riche ${ }^{32}$, il offre de belles perspectives en matière de conservation préventive. L'étude exhaustive de cette collection textile exceptionnelle ne constitue qu'un aspect du projet de plus grande ampleur qui vise à recontextualiser la nécropole gallo-romaine des Martres-de-Veyre dans son ensemble.

\section{Notes}

1. Arch. dép. Puy-de-Dôme, 19 J 20, lettre à Auguste Audollent du 20 novembre 1911.

2. Parmi lesquels J.-B. Bouillet, J.-E. Kuhn, P. P. Mathieu, F. Vazeilhes, E. Vimont, les Dr Charvilhat, Dourif et l'Héritier.

3. Programme ArchéoMartres, financé par la Maison des sciences de l'homme de ClermontFerrand; ce programme dépasse le cadre de la collection textile et envisage d'autres pistes de recherche. Principaux partenaires : université Clermont-Auvergne, musée Bargoin (Clermont métropole), Inrap. Les auteures remercient chaleureusement Sophie Desrosiers pour son soutien au projet par le dépôt de ses archives au musée et pour sa relecture attentive de ces lignes. Elles remercient également Bertrand Dousteyssier (MSH de Clermont-Ferrand, plateforme IntelEspace) et Marion Dacko (EA 1001-CHEC), membres du projet. Contact : [catherine.breniquet@uca.fr].

4. Auguste Audollent, « Les tombes de Martres-de-Veyre ", Man, 96, 1921, p. 161.

5. Auguste Audollent, « Les tombes galloromaines à inhumation des Martres-de-Veyre (Puy-de-Dôme) ", Mémoires présentés à l'Académie des inscriptions et belles lettres, 13, 1923, p. 9.

6. Datation confirmée par la présence de monnaies de Nerva, Trajan, Hadrien et Antonin, A. Audollent, ibid., p. 12-13, et plus récemment par la dendrochronologie de 170 apr. J.-C.; François BLONDEL, "Un cercueil en sapin de l'époque gallo-romaine parfaitement conservé découvert aux Martres de Veyre (Puy-de-Dôme) ", in Philippe Bet et Bertrand Dousteyssier (éd.), Éclats arvernes, fragments archéologiques ( $I^{e r}-V^{e}$ siècle apr. J.-C.), Clermont-Ferrand, Presses universitaires Blaise-Pascal, 2014, p. 254-255. 
7. Jean-Baptiste Bouillet, Musée de ClermontFerrand (Puy-de-Dôme), 1861, p. 105-106.

8. Inhumations en cercueil ou en pleine terre, avec une position du corps variable, et incinérations associées dans le même espace funéraire se retrouvent ailleurs dans le monde gallo-romain, Frédérique Blaizot et al., «Annexe 1. Les méthodes de l'archéo-anthropologie funéraire », in Christian Goudineau (dir.), Rites funéraires à Lugdunum, Paris, Errance, 2009, p. 211-215.

9. L'inventaire par tombe donné par Audollent est susceptible de modification en raison du projet en cours.

10. A. Audollent, « Les tombes galloromaines...», op. cit.

11. En réalité, une publication détaillée de ces premiers travaux était prévue, mais, faute de temps, elle n'a pas pu voir le jour. Audollent est alors allé au plus vite, piochant çà et là les principaux éléments, d'où certaines approximations.

12. A. Audollent, « Les tombes galloromaines... », op. cit., p. 19, note 1 .

13. Auguste Audollent, « Nouvelles fouilles aux Martres-de-Veyre », Bulletin historique et scientifique de l'Auvergne, 1922, p. 206-264, p. 19.

14. A. Audollent, « Les tombes des Martes-deVeyre », op. cit., p. 161-164, et Notes and Querries, 31, 1921, p. 530-531.

15. A. Audollent, « Les tombes galloromaines... », op. cit., p. 43.

16. Charles PAGÈs, "Étude technique sur les tissus découverts dans les sépultures galloromaines des Martres-de-Veyre », Mémoires présentés à l'Académie des sciences et belles lettres, 13, 1923, p. 331-384.

17. On cherchera à confirmer l'absence de coton, car cette fibre est attestée ailleurs à la même époque : Nathalie ScHLucK et al., «Un fragment de toile en coton découvert dans le frigidarium d'une villa gallo-romaine à Damblain (Vosges, France) », Archaeological Textiles Review, 54, 2012, p. 52-56; Dominique Dussot et al., « La sépulture gallo-romaine de Fontivielle (Vareilles, Creuse) », Aquitania, 18, 2001-2002, p. 157-192 (notamment l'annexe 1 : Alexandra Lorouin et Christophe Moulhérat : «Étude des vestiges textiles de la sépulture gallo-romaine de Fontvielle à Vareilles (Creuse) ».

18. Pierre Pardou Mathieu, «Des colonies romaines et des voies romaines en Auvergne, et principalement de celles qui ont donné naissance à Clermont ", Annales de l'Académie des sciences, belles-lettres et arts de Clermont-Ferrand, XXIX, 1856, p. 362.

19. La soie et l'or sont toutefois attestés ailleurs à l'époque gallo-romaine (à Naintré par exemple, à une date sensiblement plus récente : Isabelle BÉDAT et al., « Two gallo-roman graves recently found in Naintré (Vienne, France) ", in Frances Pritchard and John Peter Wild (éd.), NESAT VII. Textile Symposium in Edinburgh 5th-7th May 1999, Oxford, Owbow Book, p. 5-11; Sophie DesRosiers et Alexandra Lorquin, " Gallo-Roman archaeological textiles found in France », in Lise BENDER JøRGENSEN et Christina RinALdo (éd.), Textiles in European archaeology. Report from the 6th NESAT Symposium, 7-11th May 1996 in Borås, Göteborg, 1998, p. 58.

20. «Au lieu de décombrer le monument et de l'enlever tout d'une pièce, on s'est empressé d'en arracher, par l'ouverture que la pioche avait pratiquée, tout ce qu'il contenait, mais par morceaux ", P. P. Mathieu, «Des colonies romaines et des voies romaines en Auvergne... », op. cit., p. 362.

21. Witold Nowik et al., "The analysis of dyestuffs from First- to Second Century textile artefacts in the Martres-de-Veyre (France) excavations ", Archaeometry, 47-4, 2005, p. 835-848. Des traces bleutées sont également répertoriées sur d'autres fragments textiles.

22. Pierre-François Fournier, "Patron d'une robe de femme et d'un bas gallo-romain trouvés aux Martres-de-Veyre ", Bulletin historique et scientifique de l'Auvergne, LXXVI, 1956, p. 203.

23. Hero GRANGER-TAYLOR, comm. pers. La "ceinture" des Martres-de-Veyre s'apparente en effet à d'autres longues bandes découvertes à Masada (par ex., le numéro d'inventaire 92-113/3). L'ensemble est en cours de publication par H. Granger-Taylor dans le catalogue des textiles de Masada (Masada final reports, Israel Exploration Society).

24. A. Audollent, « Les tombes galloromaines... », op . cit., pl. $\mathrm{X}, \mathrm{n}^{\circ} 3$. Un parallèle est connu à l'âge du Fer (VIII ${ }^{\mathrm{e}}-\mathrm{vI}^{\mathrm{e}}$ siècles av. J.-C.) à Vedrette di Ries dans les Alpes italiennes, Karina Grömer, The art of Prehistoric textile making. The development of craft traditions and clothing in Central Europe, Vienna, Natural History Museum, 2016, fig. 215 , p. 388.

25. Archives inédites de S. Desrosiers déposées au musée Bargoin en 2016. À l'occasion d'une opération de conservation en 1996-1997, rapidement décidée par la municipalité et le musée de Clermont-Ferrand du fait de la présence possible de mites dans les collections, l'ensemble des fragments avait été analysé par S. Desrosiers, S. Desrosiers et A. LoRQUin, « Gallo-Roman archaeological textiles... », op. cit., en donnent une brève mention, p. 54-56. L'exploitation de ce fonds inédit est l'un des axes du projet ArchéoMartres. Les collections du musée ont également été vues 
par Hero Granger-Taylor dans les années 1980 et par Fabienne Médard dans les années 2000 (non publiés).

26. A. Audollent, « Les tombes galloromaines... », op. cit., p. 13.

27. Geneviève Roche-Bernard et Alain FERDIÈre, Costumes et textiles en Gaule romaine, Paris, Éditions Errance, 1993, p. 14.

28. W. Nowik et al., "The analysis of dyestuffs from First- to Second Century textile artefacts in the Martres-de-Veyre (France) excavations ", op. cit.

29. Examinées au début du $x^{e}$ siècle par un certain Col, membre du laboratoire de botanique de la faculté des sciences de Clermont-Ferrand, A. Audollent, « Les tombes gallo-romaines... », op. cit., p. 34-35.

30. Carol VAN DrieL-MurraY, « A set of Roman clothing from les Martres-de-Veyre, France ", Archaeological textile Newsletter, 28, 1999, p. 1.

31. Ne serait-ce que pour les tuniques, les parallèles se font avec la tunique découverte à
Bourges dans la nécropole du Fin-Renard (Alain FERDIÈRE, « Le travail du textile en Région Centre de l'Âge du Fer au Haut Moyen Âge. / Textile working in the "Région Centre " from the Iron Age to the early Middle Ages ", Revue archéologique du Centre de la France, 23-2, 1984, p. 266, fig. 55), mais aussi avec les pièces orientales (Orit Shamir, « Textiles and garments from Qumran - Chalcolithic and Roman Periods ", in Jan Gunneweg, Charles Greenblatt et Annemie Adriaens (éd.), Bio- and material cultures at Qumran, Stuttgart, Fraunhofer IRB Verlag, 2006, p. 285-296) ou égyptiennes (Dominique CARDon et al., " How were they dressed? Fragments of clothing found at Didymoi : case studies ", in Hélène Cuvigny (éd.), Didymoi. Une garnison romaine dans le désert oriental d'Égypte, t. 1 : Les fouilles et le matériel, Le Caire, Ifao, 2011, p. 273-362).

32. A. Audollent, " Les tombes galloromaines... », op. cit., p. 54. 